\title{
Hábitos que impiden la adaptación y adopción de la lectura en estudiantes de administración de empresas
}

\section{Adverse habits and barriers for the adaptation and adoption of reading}

\author{
Edward Fabián Moreno - Mendoza ${ }^{1}$, María Fernanda Coronel - Arenas ${ }^{2}$ \\ ${ }^{1}$ Corporación de Gestión Empresarial, Investigativa y de Proyectos (CORGEINP), Colombia, ORCID: \\ https://orcid.org/0000-0002-8558-6551 Email: morenomendozaef@gmail.com \\ ${ }^{2}$ Corporación de Gestión Empresarial, Investigativa y de Proyectos (CORGEINP), Colombia \\ ORCID: https://orcid.org/0000-0001-7543-2236 Email: mariacoronelar@gmail.com
}

Cómo citar: Moreno - Mendoza, E. F., \& Coronel - Arenas, M. F. (2020). Hábitos que impiden la adaptación y adopción de la lectura en estudiantes de Administración de Empresas. Revista Científica Profundidad Construyendo Futuro, 9(9), 2-9. https://doi.org/10.22463/24221783.2457

Recibido: 15 de Enero de 2018 / Aprobado: 22 de Febrero de 2018

\begin{abstract}
Resumen
A través del tiempo, la lectura se ha forjado como una actividad indispensable para el ser humano, convirtiéndose en una herramienta indiscutible para el desarrollo de nuevos conocimientos y alternativa de aprendizaje en cualquier contexto, de allí, su importancia dentro de la formación educativa superior para la adquisición de competencias que generen personas más integras capaces de afrontar cualquier proceso académico y profesional. En consonancia a lo descrito, el estudio busca identificar los hábitos que impiden la adaptación y adopción de la lectura en estudiantes del programa de Administración de Empresas de la Universidad Francisco de Paula Santander Ocaña, conociendo de esta manera, las barreras académicas, sociales y económicas que obstaculizan las prácticas lectoras. La metodología de la presente investigación se enmarca dentro de un enfoque cuantitativo de tipo descriptivo, usando como técnica "la encuesta" aplicada a una muestra de 186 estudiantes. Los resultados obtenidos demuestran que factores como la ocupación laboral y la procrastinación se consolidan como hábitos adversos que inhiben el fomento de la lectura en universitarios.
\end{abstract}

Palabras claves: Educación superior, entorno, hábitos, Lectura.

\begin{abstract}
Over time, reading has been forged as an indispensable activity for human beings, becoming an indisputable tool for the development of new knowledge and alternative learning in any context, hence its importance within higher education training for the acquisition of skills that generate more integrated people capable of facing any academic and professional process. In line with what has been described, the study seeks to identify the habits that prevent the adaptation and adoption of reading in students of the Business Administration program of the Universidad Francisco de Paula Santander Ocaña, thus knowing the academic, social and economic barriers that hinder reading practices. The methodology of the present investigation is framed within a quantitative approach of descriptive type, using like technique "the survey" applied to a sample of 186 students. The results obtained show that factors such as employment and procrastination are consolidated as adverse habits that inhibit the promotion of reading.
\end{abstract}

Key words: Higher education, environment, habits, reading.

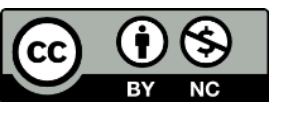

* Autor para correspondencia

Correo electrónico: josalcedo@unisalle.edu.co

La revisión por pares es responsabilidad de la Universidad Francisco de Paula Santander Ocaña

Artículo bajo licencia CC BY-NC (https://creativecommons.org/licenses/by-nc/4.0/) 


\section{Introducción}

La lectura, hoy día se ha consolidado como una actividad que forja sociedades, no solo con intelecto maduro y crítico, sino que a su vez, incide en el desarrollo personal de cada individuo, así mismo, tiene una alta influencia en el crecimiento de los ámbitos culturales, políticos y económicos a partir de la generación de habilidades y competencias basadas en el conocimiento (Flores, 2016), de allí, la gran necesidad de promover la formación de profesionales orientados a los hábitos de la lectura que faciliten la comprensión y obtención de todo tipo de información útil para la construcción de un tejido social más robusto que logre suplir las necesidades que pueda presentar.

Acorde a lo manifestado por Rovira y López (2017) "En las sociedades del conocimiento, un elemento central es la capacidad para identificar, producir, tratar, transformar, difundir y utilizar la información con vistas a crear y aplicar los conocimientos necesarios para el desarrollo humano" (p. 388). No obstante, a nivel nacional, es posible que se desconozca la repercusión que pueda desplegar la carencia de hábitos lectores, de hecho, datos demuestran que en Colombia, según el (Departamento Administrativo Nacional de Estadística [DANE], 2017) existe solo un promedio de 5.1 libros leídos por persona anualmente, cifra que es una de las más bajas a nivel internacional, en comparación con países como Canadá donde se observa una ponderación de 20 libros leídos por persona al año, igualmente en Finlandia con un equivalente anual de 17 libros leídos por persona, estados que ostentan economías totalmente sustentables a nivel mundial (Canal Capital, 2018).
Partiendo de la antepuesto, se asume que el actual panorama, en el que prima la globalización y el avance de las tecnologías de la información y la comunicación (TIC), factores como la educación y la lectura, constituyen los pilares estratégicos del progreso de las naciones y, por consiguiente, el mejoramiento de la calidad de vida de sus habitantes (Galán, 2015).

En consonancia a todo lo descrito, el estudio pretende identificar los hábitos que dificultan la adaptación y adopción de la lectura en el estilo de vida como cultura social y personal, siendo la investigación de gran relevancia en la medida en que permite conocer las barreras académicas $\mathrm{y}$ socioeconómicas que impiden la lectura en los estudiantes del programa de Administración de Empresas de la Universidad Francisco de Paula Santander Ocaña (UFPSO), dejando en evidencia las razones reales que imposibilitan el fortalecimiento de prácticas lectoras, además de ser un referente para futuros estudios.

Entonces, para recapacitar, mejorar y accionar hacia un mundo mejor, es necesario plantear aquellos puntos que obstruyen está transformación, ya que detrás de una acción esta una concepción y de esta se desprenden unas costumbres que solo podrán ser reformadas por medio del seguimiento y direccionamiento de los más jóvenes hacia un estilo de vida que los hará idóneos para las nuevas exigencias del entorno, determinando aquellos hábitos que dificultan la formación integral de las nuevas generaciones y a su vez el mejoramiento continuo de las mismas.

\section{Marco Teórico}

La lectura, además de ser una herramienta para el desarrollo personal y económico de 
una sociedad, es una actividad que permite a las personas crear y almacenar una serie de información y vocabulario que pueden ser determinantes en su interacción social, ya que, al presentarse esta dinámica entre el sujeto y el texto, se construye una relación que crea competencias y actitudes propicias para su praxis. A continuación, se plasman algunas teorías que fundamentan el estudio.

\subsection{Teoría lineal e interactiva}

La teoría lineal se enfoca en la primera etapa que figura la comprensión textual, concibiéndose como un proceso perceptual directo, secuencial y ordenado, donde los lectores decodifican símbolos gráficos, que se traducen en códigos orales. Se aduce que el material escrito es, esencialmente, la comprensión del habla, originada en la mente del lector, por tanto, permite plantear una concepción de lectura en la cual esta transforma los símbolos gráficos en significados, resaltando los procesos de reconocimiento de la palabra escrita (Gough, 1994; Laberge y Samuels, 1994; Linuesa y Domínguez, 1999; Parodi, 1999) citados en (Makuc y Larrañaga, 2014). Por su parte, la teoría interactiva agrupa los sistemas perceptivos y cognitivos en los que se genera un proceso de forma descendente $y$ ascendente por parte del lector, procesando el texto escrito desde sus conocimientos, activados durante la lectura y los datos contenidos en el texto, formando un lector activo capaz de procesar la información del texto a partir de sus propios conceptos procedentes del discernimiento $\mathrm{y}$ experiencias propias (van Dijk y Kintsch, 1983; Goodman y Goodman; 1994; Colomer y Camps, 1996; Rumelhart, 1997; De Vega, 1998, 2004; Parodi, 2005, 2007) citados en (Makuc y Larrañaga, 2014).

\subsection{Teoría literaria}

Esta teoría emerge como una disciplina cuya finalidad es el surgimiento de un método científico de análisis de la obra literaria, la cual implica la facultad imaginativa $y$ caracterización de personajes y situaciones a partir de la lectura y vinculación con sucesos de la vida real, adquiriendo competencias de comprensión implícitas en la comparación de textos literarios con base a acciones cotidianas, promoviendo de esta manera la capacidad de análisis crítico, creación y composición de argumentos y fundamentaciones propias del lector tomando como referencia la vivencia de hechos y fenómenos producidos en su entorno social (Martínez, 2018).

\subsection{Teoría del enfoque sociocultural de Vygotsky}

Mantiene una perspectiva evolutiva, explicando el desarrollo del sujeto por medio de interacciones y experiencias, como lo afirma Vygotsky un comportamiento sólo puede ser entendido si se estudian sus fases, su cambio, es decir; su historia. Este enfoque le da prioridad al análisis que dilucida el autor en la comprensión y forma de los procesos psicológicos por medio del entendimiento de los sucesos que se presentan e intervienen en su desarrollo. Es por esto que Vygotsky consideró los efectos de la interrupción y las intervenciones sobre ellos; dando lugar a las variantes del análisis genético: el método genético-comparativo y el método experimental-evolutivo (Citado en Carrera, 2001).

\section{Metodología}

La presente investigación se enmarcó dentro del enfoque cuantitativo de tipo descriptivo, que según Hernández, Fernández 
y Baptista (2014) está orientado a la descripción de características y rasgos de hechos, fenómenos y/o situaciones, para posteriormente ser analizados, tal como el cometido que tiene la investigación, cuyo objetivo es la identificación de los hábitos que dificultan la adaptación y adopción de la lectura en el estilo de vida como cultura social y personal de los estudiantes del programa de Administración de Empresas de la Universidad Francisco de Paula Santander Ocaña (UFPSO), siendo estos últimos la población objeto de estudio, los cuales conforman una muestra total de 619 estudiantes; al ser una muestra relativamente grande se procedió a la aplicación de la formula finita:

$$
\mathrm{n}=\frac{(\mathrm{Z})^{2} * N * P * Q}{\mathrm{E}^{2}(N-1)+(Z)^{2} * P * Q}
$$

Donde:

$\mathbf{n}=$ Muestra

$\mathbf{N}=$ Población de estudio $=619$

$\mathbf{Z}=$ Nivel de confianza $=95 \%=1.96$

$\mathbf{P}=$ Proporción de aceptación $=50 \%=0.5$

$\mathbf{Q}=$ Proporción de rechazo $=50 \%=0.5$

$\mathbf{E}=$ Error de estimación $=6 \%=0.06$

Entonces:

$$
\begin{gathered}
\mathrm{n}=\frac{(1.96)^{2} * 619 * 0.5 * 0.5}{0.06^{2}(619-1)+(1.96)^{2} * 0.5 * 0.5} \\
\mathrm{n}=186 \text { Estudiantes }
\end{gathered}
$$

Tomando en cuenta la naturaleza de la investigación, se optó como técnica para la recolección de datos la aplicación de la encuesta y como instrumento el cuestionario, estructurado a partir de una serie de preguntas orientadas a conocer los hábitos que impiden la adaptación y adopción de la lectura. Para el procesamiento de la información, una vez recolectada, se procedió a tabular los datos obtenidos, seguidamente, a través del software estadístico y de cálculo Excel se condenso la información ilustrándose por medio de tablas y gráficas, para consecutivamente desarrollar un análisis descriptivo de cada una, dando cumplimento al estudio.

\section{Resultados}

Tras el tratamiento de los datos adquiridos con la aplicación de los instrumentos para la recolección de información, se reconocen los hallazgos sobre la identificación de los hábitos que dificultan la lectura en los estudiantes de Administración de Empresas de la UFPSO, anexados, a continuación.

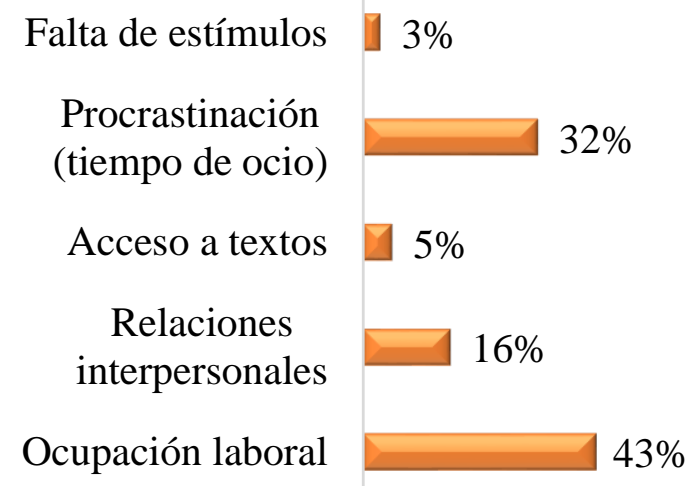

Figura 1. Hábitos que impiden la lectura. Fuente: Autores del artículo.

A partir de los compendios estadísticos se observa que en un $43 \%$ los estudiantes de Administración de Empresa de la UFPSO, manifiestan que se les dificulta adquirir prácticas lectoras dado a sus ocupaciones laborales que, al unirse a sus horarios académicos, abarcan por completo su tiempo diario, generando cansancio para la realización de otras actividades. 
Por otra parte, se evidencia en un $32 \%$ estudiantes que no conciben pericia de lectura por hábitos de procrastinación o excesiva inversión en tiempo de ocio, expresando que en su cotidianidad suelen estar inmersos en la utilización de redes sociales, juegos online, horas extensas en programas televisivos o contenido streaming, entre otros; así mismo, un $16 \%$, indica que prefiere invertir su tiempo libre con amigos, familiares o personas cercanas (relaciones interpersonales).

Finalmente, bajo una ponderación del 5\% existen estudiantes que expresan no leer, por falta de acceso a textos, aludiendo que en sus casas no presencian material de lectura o no hay disponibilidad de bibliotecas públicas, bien sea por su inexistencia o lejanía, en otros casos, se da por la carencia de ingresos económicos que posibilite la compra de libros, revistas, periódicos, etc., e incluso la asequibilidad a herramientas tecnológicas que permitan la obtención de textos digitales. Por último, en un $3 \%$ están quienes mencionan que la falta de estímulos por parte de familiares, amigos y/o docentes, hacen que pierdan el interés por la lectura.

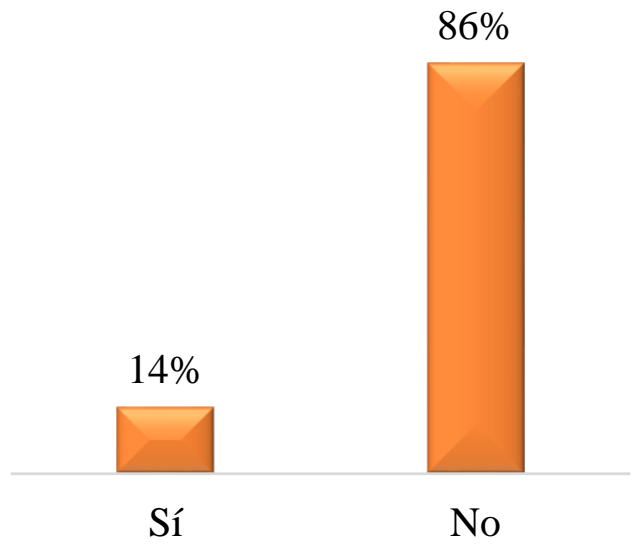

Figura 2. Conocimiento de los beneficios de la lectura. Fuente: Autores del artículo.
Según los datos obtenidos con el instrumento, se determina que la mayoría de estudiantes de Administración de Empresas de la UFPSO (86\%) desconoce los beneficios que puede aportar el hábito de la lectura, aduciendo que poco la practican; en contraste el $14 \%$ restante afirma si conocerlos, manifestando algunos de ellos: Generación de nuevo conocimiento, ampliación de vocabulario, desarrollo de pensamiento crítico y analítico, y estimulación mental.

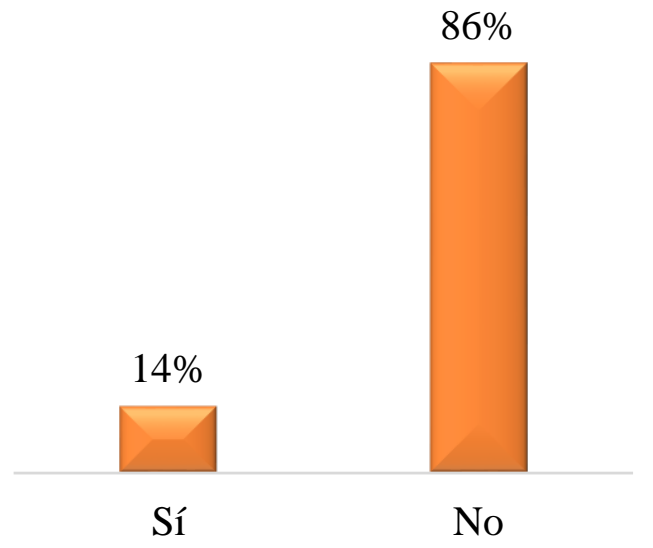

Figura 3. Inserción de estrategias para la obtención de hábitos lectores. Fuente:

Autores del artículo.

Conforme a los resultados arrojados por la encuesta, se visibiliza en un $86 \%$ la ausencia de estrategia orientadas a la adquisición de hábitos lectores, donde los encuestados enunciaron no presentar alguna preocupación por crear o promover alguna acción o compromiso que sea destinado al provecho de la lectura, ignorando los beneficios que puedan obtenerse con este hábito. No obstante, el 14\% que afirmo aplicar algún tipo de estrategia para fomentar la lectura, pronunció estipular un tiempo de 10 minutos para leer y elección de textos a fines a sus preferencias delimitando número de páginas leídas por día. 


\section{Discusiones}

De los hallazgos obtenidos con los resultados, se obtiene que los hábitos más preponderantes que dificultan la adquisición de lectura en los estudiantes del programa de Administración de Empresas de la UFPSO, son las ocupaciones laborales y la procrastinación; causando lo anterior, acorde con Barrio, Borragá, Pérez y Castro (2005) barreras que obstaculizan a los universitarios generar respuestas eficaces a las demandas del texto académico y por ende bajo rendimiento de los procesos formativos, dado que no hay un nivel de comprensión de las asignaturas contenidas en su pensum, ni la capacidad de relacionar lo visto en aulas de clase con los contextos sociales, culturales, económicos y laborales, siendo la lectura, según la teoría literaria, la única capaz de gestionar competencias de análisis crítico y creación de acciones cotidianas a partir de la adaptación y adopción de hábitos lectores.

Ahora, lo que posiblemente para muchos resultase superfluo, partiendo del supuesto que en la actualidad son muchos los estudio que evidencia los beneficios que produce la lectura, los resultados demuestran que la mayoría de los estudiantes del programa no tienen conocimiento de estos, aun cuando los mismos (beneficios) son indispensables para su desarrollo cognitivo. De hecho, Pérez, Baute y Espinoza (2018) sostienen que, en el contexto universitario la lectura es un elemento infalible, pues conforma la mayor parte del ejercicio académico, científico y de conexión con la comunidad, como ruta de entrada a la gestión de la información y el conocimiento, siendo estos dos factores ineludibles para la apropiación de las competencias profesionales y una cultura general.
Los estudiantes universitarios requieren del hábito de la lectura para realizar las múltiples tareas de aprendizaje a las que se enfrentan de manera cotidiana, a partir de las exigencias de consultar variada bibliografía física, digital y realizar búsquedas en internet y como medio para hacer un buen uso del tiempo libre. (p.181)

Finalmente, implementar estrategias que fomente y promuevan la lectura son vitales para ayudar a superar los obstáculos que imposibilitan la praxis literaria, sin embargo, para el caso planteado por la investigación, se exhibe que el estudiantado en más del ochenta por ciento, no ejecuta alternativas para la obtención de hábitos lectores, haciendo más complejo su avance. Entonces, como declaran Cardona, Osorio, Herrera y González (2018) permite entrever que aún en la educación superior subsisten problemas que evocan una apatía hacia la lectura, trayendo consigo contratiempos que pueden significar desempeños académicos regulares y serias limitaciones en la formación profesional.

\section{Conclusiones}

Tras culminar el estudio, se concluye que, contra todos los pronósticos, dado a las premisas que pudiesen suscitar sobre la responsabilidad y el nivel que demanda la obtención de una educación superior, se exhibe una prominente carencia de conductas lectoras en los estudiantes del programa de Administración de Empresas de la UFPSO, causados por hábitos que trasciende más allá del ejercicio académico, tales como: las ocupaciones laborales y la procrastinación, dejando en tela de juicio el grado de importancia que estos otorgan al desarrollo de su crecimiento profesional.

Por otro lado, dato relevante que se logra con la realización de la investigación es que 
en un $86 \%$ el estudiantado admite desconocer los beneficios que se pueden adquirir a través de la lectura, que aunque fuese posible adjudicar lo anterior como la justificación del bajo nivel de praxis lectora, en realidad no resulta una excusa valida, pues al recibir una educación universitaria, la exigencia académica connota una mayor apropiación y generación de nuevos conocimientos, que desde luego, son proporcionados por el fortalecimiento cognitivo que despliega la acción literaria.

Partiendo de lo anterior, se evidencia que ese mismo porcentaje (86\%) no lleva a cabo estrategias que permitan gestionar $y$ promover nuevas costumbres orientadas a la obtención de lectura, demostrando una vez más el desinterés que poseen los estudiantes por mejorar sus hábitos lectores. Entonces, resulta ineludible la sensibilización de acciones y compromisos encaminados hacia el ejercicio lector como alternativa para el fomento de habilidades y competencias en la construcción de profesionales con posturas más críticas y fundamentos que conciban contextualizar el aprendizaje académico con los factores sociales, económicos y culturales.

\section{Referencias}

Barrio, J., Borragá, A., Pérez, M., \& Castro, S. (2005). Potenciación de la lectura en estudiantes universitarios: Planteamientos para un reto de futuro. Revista de psicología INFAD, 91-105. Recuperado el 2018, de https://www.redalyc.org/pdf/3498/34 9832309006.pdf

Canal Capital. (2018). ¿Qué, cómo y cuánto leen los colombianos? Recuperado el 2018, de conexioncapital.co: https://conexioncapital.co/cuantoleen-los-colombianos/

Cardona, S., Osorio, A., Herrera, A., \& González, J. (2018). Actitudes, hábitos y estrategias de lectura de ingresantes a la educación superior. Revista Educación y Educadores, 482-503. Recuperado el 2018, de https://dialnet.unirioja.es/servlet/artic ulo? codigo $=6916168$

Carrera, B. (2001). Vygotsky: Enfoque sociocultural. Revista Venezolana de Educación EDUCERE, 41-44. Recuperado el 2018, de https://dialnet.unirioja.es/servlet/artic ulo? codigo $=3650617$

DANE. (2017). Encuesta Nacional de Lectura-ENLEC. Informe estadístico. Recuperado el 2018, de https://www.dane.gov.co/files/investi gaciones/boletines/enlec/presentacion -enlec-2017.pdf

Flores, D. (2016). La importancia e impacto de la lectura, redacción y pensamiento crítico en la educación superior. Revista del Instituto de Estudios en Educación Universidad del Norte, 128-135. Recuperado el 2018, de http://www.scielo.org.co/pdf/zop/n24 /n24a10.pdf

Galán, J. (2015). Promoción de lectura para estudiantes de licenciatura en gestión de negocios. Recuperado el 2018, de uv.mx:

https://www.uv.mx/epl/files/2015/08/ protocolo_roberto_galan_garcia.pdf

Hernández, R., Fernández, C., \& Baptista, M. (2014). Metodología de la investigación sexta edición. México 
D.F: McGraw-Hill. Recuperado el 2018

Makuc, M., \& Larrañaga, E. (2014). Teorías implícitas acerca de la comprensión de textos: Estudio exploratorio en estudiantes universitarios de primer año. Revista Signos-Estudios de lingüística, 29-53. Recuperado el 2018, de https://scielo.conicyt.cl/pdf/signos/v4 8n87/a02.pdf

Martínez, F. (2018). Teoría literaria. Recuperado el 2018, de memoriachilena.gob.cl:

http://www.memoriachilena.gob.cl/6 02/w3-article-93896.html

Pérez, V., Baute, M., y Espinoza, M. (2018). El hábito de la lectura: Una necesidad impostergable en el estudiante de ciencias de la educación. Revista Científica de la Universidad de Cienfuegos, 180-189. Recuperado el 2018, http://scielo.sld.cu/pdf/rus/v10n3/221 8-3620-rus-10-03-180.pdf

Rovira, Y., y López, E. (2017). La lectura en la enseñanza universitaria. Revista de Ciencias Médicas de Pinar del Río, 386-398. Recuperado el 2018, de http://scielo.sld.cu/pdf/rpr/v21n3/rpr1 3317.pdf 